\title{
PHOTOIONIZATION DYNAMICS OF SMALL MOLECULES WITH SYNCHROTRON RADIATION: STATE PREPARATION BY PEPICO AND TPEPICO
}

\author{
PAUL MARIE GUYON \\ LCAM Bat 351 and LURE Bat 209 d, Université Paris, XI 91405, Orsay, \\ Cedex, France
}

The use of synchroton radiation combined with TPEPICO experiments to study the photoionization dynamics of molecules at Orsay's synchroton radiation facility is discussed. The initial state preparation by Threshold Photoelectron Spectroscopy and final state-mass and internal energy spectroscopy by time of flight analysis of photoelectrons as well as ions in coincidence with threshold electrons is illustrated by the TPES of $\mathrm{HCl}$, the TOF TPES of $\mathrm{O}_{2}$ and the TPEPICO spectra of $\mathrm{NO}^{+}$fragments from the decay of state selected $\mathrm{N}_{2} \mathrm{O}^{+}$ions.

KEY WORDS: Photoionization, threshold photoelectron spectroscopy, TPEPICO, synchroton radiation.

\section{INTRODUCTION}

Synchroton radiation has been used in Orsay since 1975 to study molecular photoionization: direct versus autoionization processes, the unimolecular decay of vibronically excited ions and the reactivity of state selected ions. For such studies, synchrotron radiation from electron (ACO) or positron (Super ACO) storage rings present a great advantage over conventional laboratory VUV light sources in that it produces a broad continuum extending over the whole spectroscopic domain allowing rotational, vibrational and electronic excitation from valence to $\mathrm{K}$ shell electrons. These sources associated with normal incidence VUV monochromators equipped with holographic gratings of 1 or $3 \mathrm{~m}$ radius yield tunable monochromatic light with a resolution of about $1 \mathrm{meV}$ i.e. $10 \mathrm{~cm}^{-1}$ in the first order with little overlap from higher order light. These machines operating with either one or two electron bunch provide short pulses of light $1.5 \mathrm{~ns}$ (fwhm every $73.5 \mathrm{~ns}$ ) on ACO and $0.5 \mathrm{~ns}$ (fwhm every $20 \mathrm{~ns}$ ) on Super ACO. Time analysis of the excitation processes is thus made possible with a high duty cycle of the order of $10 \mathrm{Mhz}$ perfectly adapted for time of flight analysis of electrons and for coincidence studies. These unique features were exploited with specially designed time of flight spectrometers to measure:

1) the total photoionization cross section [Photoionization Efficiency curves (PIE)]; 
2) the distribution of ion vibronic states [Photoelectron Spectroscopy (PES) and Threshold Photoelectron Spectroscopy (TPES)];

3) the mass as well as the kinetic energy distributions of the ions [Time of Flight Mass Spectra (TOF-MS)];

4) the fragmentation spectrum of state selected ions [Photo Electron Photo Ion Coincidence (PEPICO) and Threshold Photo Electron Photo Ion Coincidence Spectra (TPEPICO)].

We will in this paper discuss how high resolution TOF PES can be used to study the fundamental aspects of photoionization direct and autoionization processes and how detailed informations on the dissociation of mechanisms polyatomic ions can be obtained from TPEPICO spectra.

\section{THE TPEPICO METHOD}

To study state selected fragmentation of ions the coincidence method exploits the unique property of time and energy correlation between the ejected photoelectron and the corresponding ion. The TPEPICO experiment uses time of flight analysis of both the electron and the ion.

The TPES is based on the angular discrimination of low energy electrons accelerated by a low electrostatic field of about $1 \mathrm{~V} / \mathrm{cm}$ produced when photon energy is scanned over the ion spectrum. The early methods did not provide perfect discrimination against fast electrons ejected along the field axis. ${ }^{1-3}$ Time of flight analysis of the electrons made possible with the pulsed synchrotron radiation sources allows the suppression of these unwanted electrons. The method provides a standard resolution of about $10 \mathrm{meV} .{ }^{4}$ Lately a resolution of $4 \mathrm{meV}$ was obtained. ${ }^{5}$ Ion state selection by TPES presents two advantages over conventional PES using electrostatic analysers:

a) the transmission is about three orders of magnitude greater;

b) excited vibronic states of the ion are formed in a broader energy range, actually

in the Franck-Condon gap region, states of the ion are produced by resonant autoionization which are not accessible for direct transition from the ground state molecule because of vanishingly small Franck-Condon factors.

The TOF Mass Spectrometry method as implemented in this work presents several advantages over conventional magnetic or quadrupole mass spectrometry: The use of large area channel plate detectors (i.e. $4 \mathrm{~cm}$ useful i.d.) allows the design of spectrometers free from mass as well as kinetic energy discrimination. This is essential when one wants to measure accurate branching ratios for fragmentation. The use of two acceleration field regions is necessary to optimize the spectrometer for both fragment ions kinetic energy resolution and mass resolution. In the present set up both resolutions were $50 \mathrm{meV}$ and 1/100 respectively. Finally, since optimization of collection efficiencies is of paramount importance for coincidence meas- 
urements, the high transmission of the TOF mass spectrometer (i.e. $80 \%$ ) is another advantage of the method.

The latest double electron-ion TOF coincidence spectrometer that was constructed is shown in Figure 1. Photoelectrons and ions are extracted in opposite directions at right angle from the plane defined by the jet of molecules and the photon beam. Photoelectrons accelerated by a homogeneous $1 \mathrm{~V} / \mathrm{cm}$ DC field are selected by the combined effect of their time of flight, measured with respect to the photon pulse arrival time, and by angular discrimination. The threshold electrons reach the detector in a time less than the photon pulse period (i.e. $\tau=120 \mathrm{~ns}$ on super ACO when two positron bunch are stored). Energetic electrons arrive sooner or later depending whether they are emitted forward or backward with respect to the detector direction. TOF analysis can be done to study electron energy distribution or a delayed time gate can be applied to select threshold electrons for TPEPICO measurements.

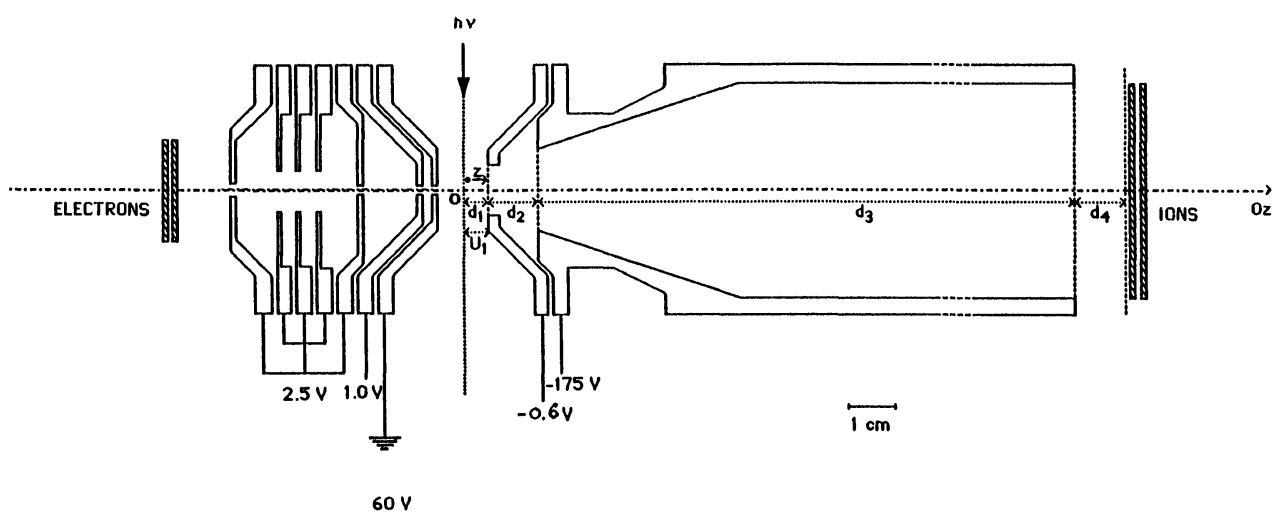

Figure 1 Double electron, ion coincidence time of flight spectrometer.

To record TPEPICO spectra an ion extraction pulse of about $60 \mathrm{~V} / \mathrm{cm}$ is triggered by the threshold electron signal. The coincident ion is post accelerated by a $175 \mathrm{~V} / \mathrm{cm}$ DC field and fly in the field free $14 \mathrm{~cm}$ long region before reaching the detector. The coincident ion time of flight is thus measured with respect to that of the threshold electron. Metastable ions which dissociate in flight in any of the to acceleration regions arrive at the detector in a longer time so that analysis of the peak shape gives the ion lifetime. The limit of sensitivity is $\tau=200 \mathrm{~ns}$ with the present set up. ${ }^{6}$

\section{THE HCI TPES: DIRECT IONIZATION, SPIN-ORBIT AND ROTATIONAL AUTOIONIZATION}

TPE spectra are usually more structured than the corresponding PES for the same energy band pass, they present also many more lines. This phenomenon has been early recognized as due to autoionization. ${ }^{7}$ Rydberg states of the molecule which lie 
(StỊun •qre) suot

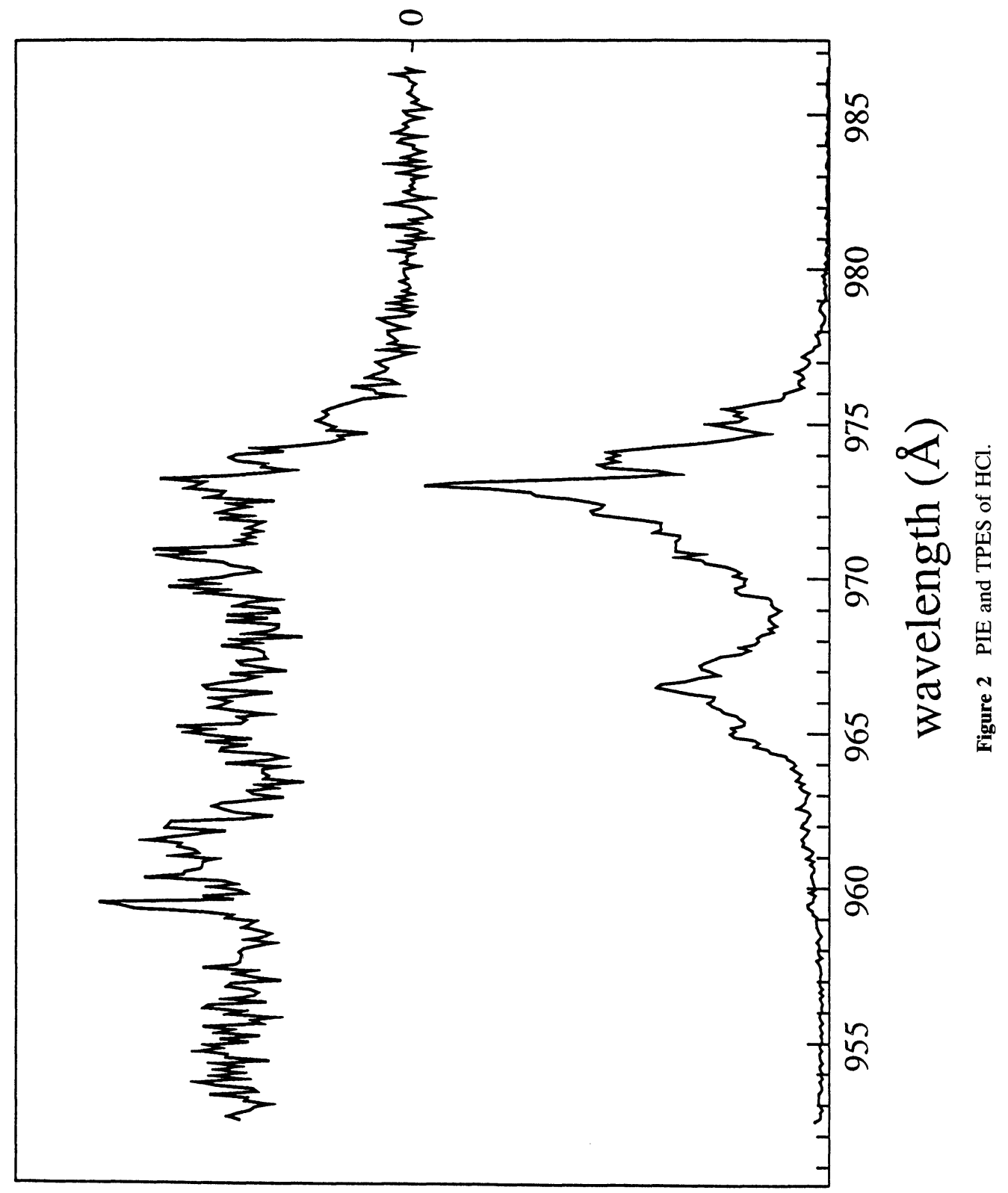

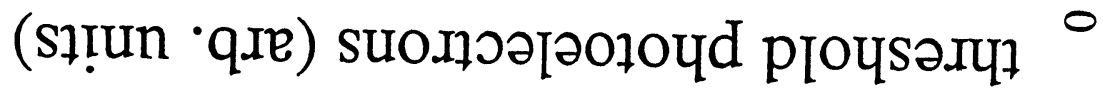


above vibronic levels of the ion but within the band pass of the analyser may autoionize to produce threshold electrons. The intensity of the lines in a TPES is thus directly affected by the occurrence of resonances. Because TPES is so simple and so efficient to produce state selected ions it is widely used for coincidence measurements. It is important to understand the spectra in order to know the quantum state of the ion thus prepared. The TPES of $\mathrm{HCl}$ presented below show that this objective can be achieved. ${ }^{8}$

Figure 2 shows the two first bands of the HCl TPES and the corresponding PIE obtained with an $0.02 \mathrm{~nm}$ photon band-width, $1 \mathrm{~V} / \mathrm{cm}$ DC acceleration field and an electron time of flight gate of $5 \mathrm{~ns}$ width. The TPES is quite different from the PES of Natalis et al. ${ }^{9}$ which was obtained at about the same overall resolution:

1) The $X^{2} \Pi_{3 / 2}$ band is about twice as intense as the ${ }^{2} \Pi_{1 / 2}$ although the two states present the same degeneracy and have the same intensity in the PES.

2) The peaks are structured and some of this structure is also found in the PIE spectrum.

3) The ${ }^{2} \Pi_{3 / 2}$ peak is broader and tails at lower photon energy.

Such differences are common for most TPES and are understood as follows:

Each rovibronic state of the ion excited within the photon energy bandpass is a limit of Rydberg series. A number of these bound levels as well as continuum levels above the limits are excited. The latter contribute to the direct ionization channel whereas the former have two different contributions. The highest $n$ levels lying within $1 \mathrm{meV}$ of the limit are metastable with respect to predissociation or preionization, they are field ionized, thus contributing to the direct ionization channel. This lowers the apparent ionization potential by $1 \mathrm{meV}$ (this value is proportional to the square root of the field). The lower $n$ states can autoionize to any of the lower lying states of the ion and the associated threshold electrons constitute the resonance contribution to the TPES.

In order to perform a quantitative analysis of the spectrum one needs to have a model to represent each category of processes. Here the direct ionization signal due to the ejection of $p \pi$ electrons was calculated for each rotational level $J^{\prime \prime}$ of the molecule with the selection rule: $\left|J^{\prime \prime}-J^{+}\right| \leqq 1 / 2$ which assumes that none of the photon angular momentum is given to the rotating nuclei. The small contribution of field ionization is ignored. Autoionization has two contributions: the levels converging to ${ }^{2} \Pi_{1 / 2}$ autoionize to ${ }^{2} \Pi_{3 / 2}$ by spin orbit interaction and slower rotational autoionization to the same electronic limit $X{ }^{2} \Pi_{1 / 2}$ is ignored. Fitting of the experimental data shows that as many as two quanta of angular momentum could be exchanged between the Rydberg $d$ type electron and the ion core with the selection rule: $\left|J^{\prime \prime}-J^{+}\right| \leqq 7 / 2$. This contribution to the lowest energy ${ }^{2} \Pi_{3 / 2}$ peak cancels when one reaches the upper ${ }^{2} \Pi_{1 / 2}$ state for which it becomes the direct ionization continuum. There is no spin-orbit autoionization to ${ }^{2} \Pi_{1 / 2}$ and this explains the ratio of about 2 and the difference in widths between the two bands. The high $J$ Rydberg levels converging to ${ }^{2} \Pi_{3 / 2}$ autoionize by rotational coupling to the energetically accessible ${ }^{2} \Pi_{3 / 2} J^{+}$states. This process is slower than $5 x 10^{-9} \mathrm{~s}$, the experimental photoelectron time window so that this process does not contribute to our TPES, but 


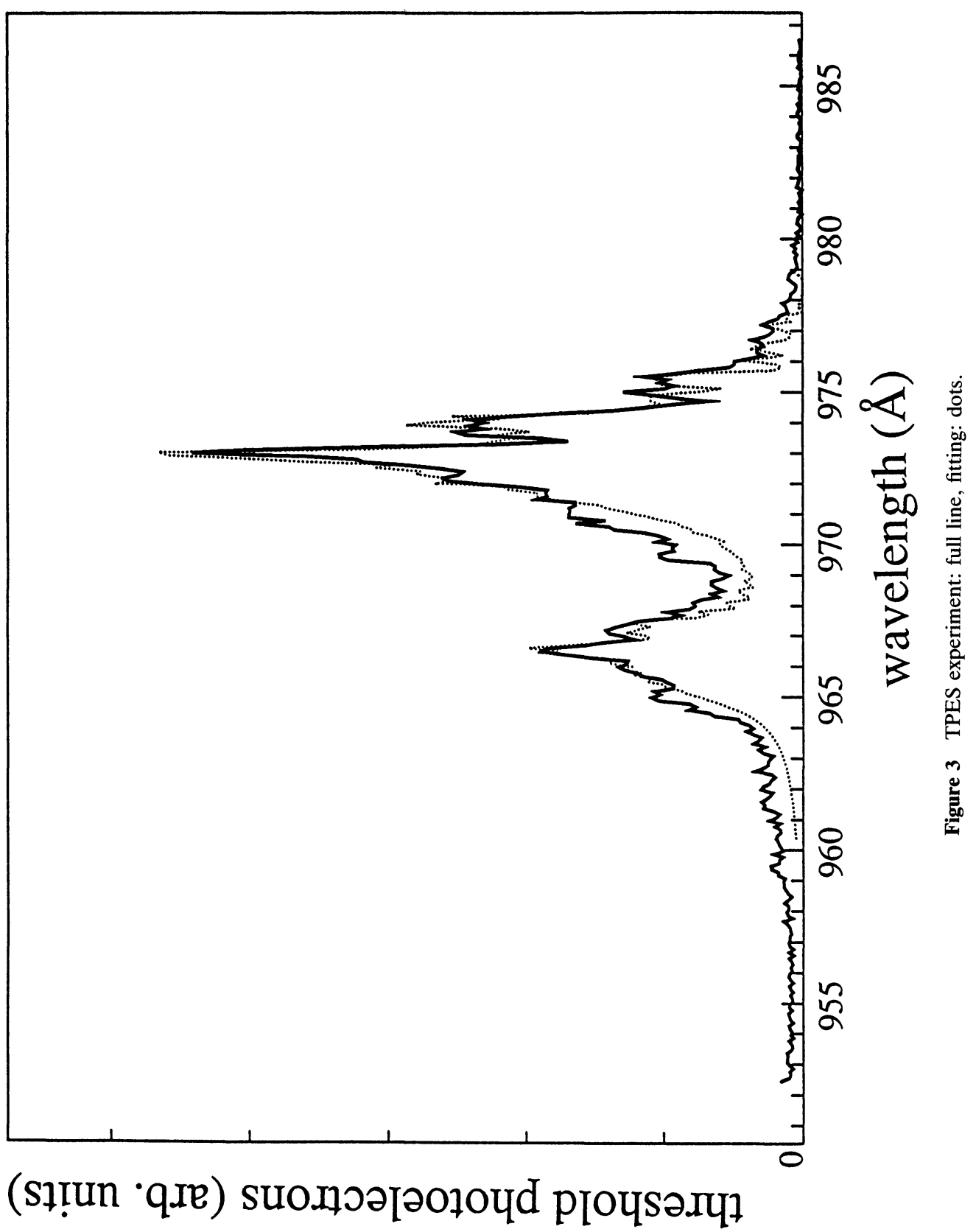


adds a low energy tail to the PIE as it has been recognized long ago. The experimental results and a model Monte-Carlo simulation are compared in Figure 4.

\section{ELECTRONIC AUTOIONIZATION IN O 2}

Photoionization of oxygen molecule near the ionization threshold shows intense autoionization peaks superimposed on a weak continuum. Some of these structures. assigned to ${ }^{3} \Pi_{u}$ Rydberg series converging to $\mathrm{O}_{2}^{+} a^{4} \Pi_{u}$ are seen to be split into several fine structure spin orbit components in the high resolution PIE. ${ }^{10}$ These series autoionize to $\mathrm{O}_{2}+\mathrm{X}^{2} \Pi_{g}$ ground state and the vibrational branching ratio is consistent with the model of Bardsley and Smith for electronic autoionization. ${ }^{11,12}$ We were able to resolve the two $\Omega^{+}=1 / 2$ and $\Omega^{+}=3 / 2$ spin-orbit components of the ground state ion vibrational levels and investigated the variation of the ${ }^{2} \Pi_{g 1 / 2} /$ ${ }^{2} \Pi_{g 3 / 2}$ branching ratio upon selective excitation of the $\mathrm{F}_{1}(\Omega=2), \mathrm{F}_{2}(\Omega=1)$ and $\mathrm{F}_{3}(\Omega=0)$ spin-orbit components of the $\mathrm{H}(v=0)$ and $\mathrm{H}(v=2)$ series. The experiment was done on Super ACO with a 3 m normal incidence monochromator and the double electron ion time of flight coincidence analyser shown in Figure 1. A free expansion jet was adapted to cool the molecules. Photoelectron peaks separated by about $5 \mathrm{meV}$ at energies as low as $10 \mathrm{meV}$ were for the first time resolved. ${ }^{5}$

The electron time of flight spectra obtained upon excitation of the $\mathrm{H}(v=0) \mathrm{F}_{1}, \mathrm{~F}_{2}$ and $\mathrm{F}_{3}$ components are shown in Figure 4 . They are roughly symmetrical with respect to $\mathrm{T}(0)$ the time of flight of the zero energy electrons because of the contribution of both the forward and backward ejected electrons. If we consider only the forward peaks associated with $\mathrm{O}_{2}^{+}+v^{+}=1$ and 2 , one observes that they are split into their two spin-orbit components $\Omega^{+}=1 / 2$ and $3 / 2$. The variation in the ${ }^{2} \Pi_{g 1 / 2}:{ }^{2} \Pi_{g 3 / 2}$ branching ratio with excitation energy is dramatic and clearly indicates a strong correlation between $\Omega$ and $\Omega^{+}$, the projections of the total angular momentum on the molecular axis. We do not consider the molecular rotation which can be neglected since the rotational spacing is much less than the fine structure level separation. The experimental results which have been fitted with a Monte Carlo simulation are presented in Table 1 .

The correlation between the high values of $\Omega$ in the resonance state and the continuum state is shown to reflect a strong selectivity. Among the two $\left(\pi_{g} k \sigma_{u}\right)$ $\left(\pi_{g} k \delta_{u}\right)$ continua accessible according to Kronig's selection rules for electronic autoionization, the $\mathrm{H}^{3} \Pi_{u}$ Rydberg series of the oxygen molecule decay almost entirely into $\left(\pi_{g} k \sigma_{u}\right)$. This in turn means that the bi-electronic integrals of the type $\left(\pi_{u} \sigma_{g} 1 / r_{12} \pi_{g} k \sigma_{u}\right)$ are much greater than $\left(\pi_{u} \sigma_{g} 1 / r_{12} \pi_{g} k \delta_{u}\right)$.

\section{FRAGMENTATION OF TRIATOMIC ION: $\mathrm{N}_{2} \mathrm{O}^{+}$A STATE}

$\mathrm{N}_{2} \mathrm{O}^{+} \mathrm{A}{ }^{2} \Sigma^{+}$state dissociation is one of the most studied fragmentation of a polyatomic ion. This first excited state lies some $3 \mathrm{eV}$ above the ground ${ }^{2} \Pi$ state; they both have the same geometry as the ground state molecule. Figure 5 shows schematic cuts of the potential energy surfaces for linear $\mathrm{N}_{2} \mathrm{O}^{+}$along the 


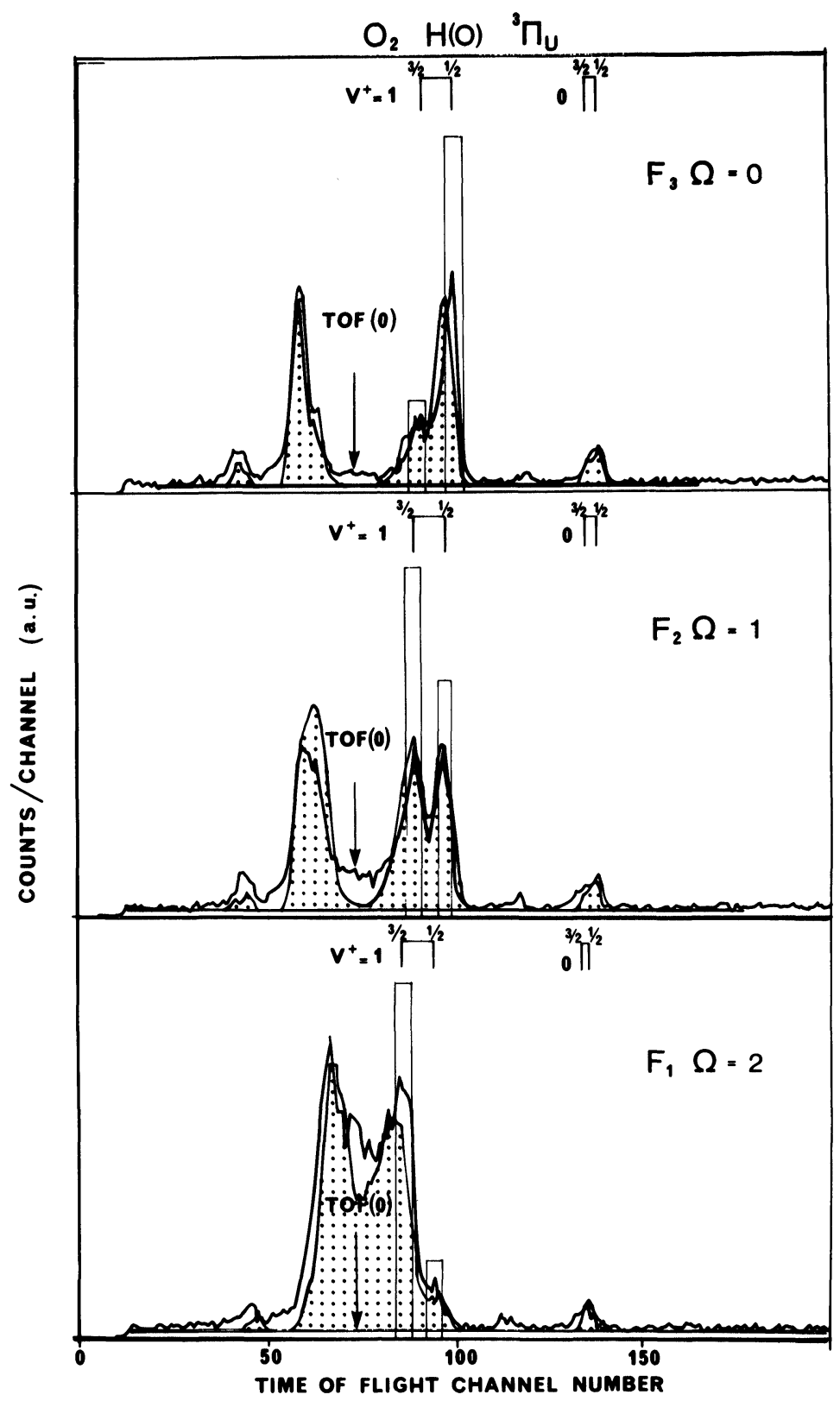

Figure 4 TOF Photoelectron spectra of $\mathrm{O}_{2}$ obtained upon excitation of the $\mathrm{H}(0) \leqq X(0,0)$ band at (a) $\Delta=100.38 \mathrm{~nm}$, (b) $\Delta=100.31 \mathrm{~nm}$, (c) $\Delta=100.26 \mathrm{~nm}$. Histograms represent the peak area divided by the transmission function, smooth and shaded by dots are the result of the Monte-Carlo simulation. The time of flight decreases with increasing channel number and the arrows indicate the time of flight of strictly zero energy electrons. The peaks on the left side of the arrows are due to backward ejected electrons. 


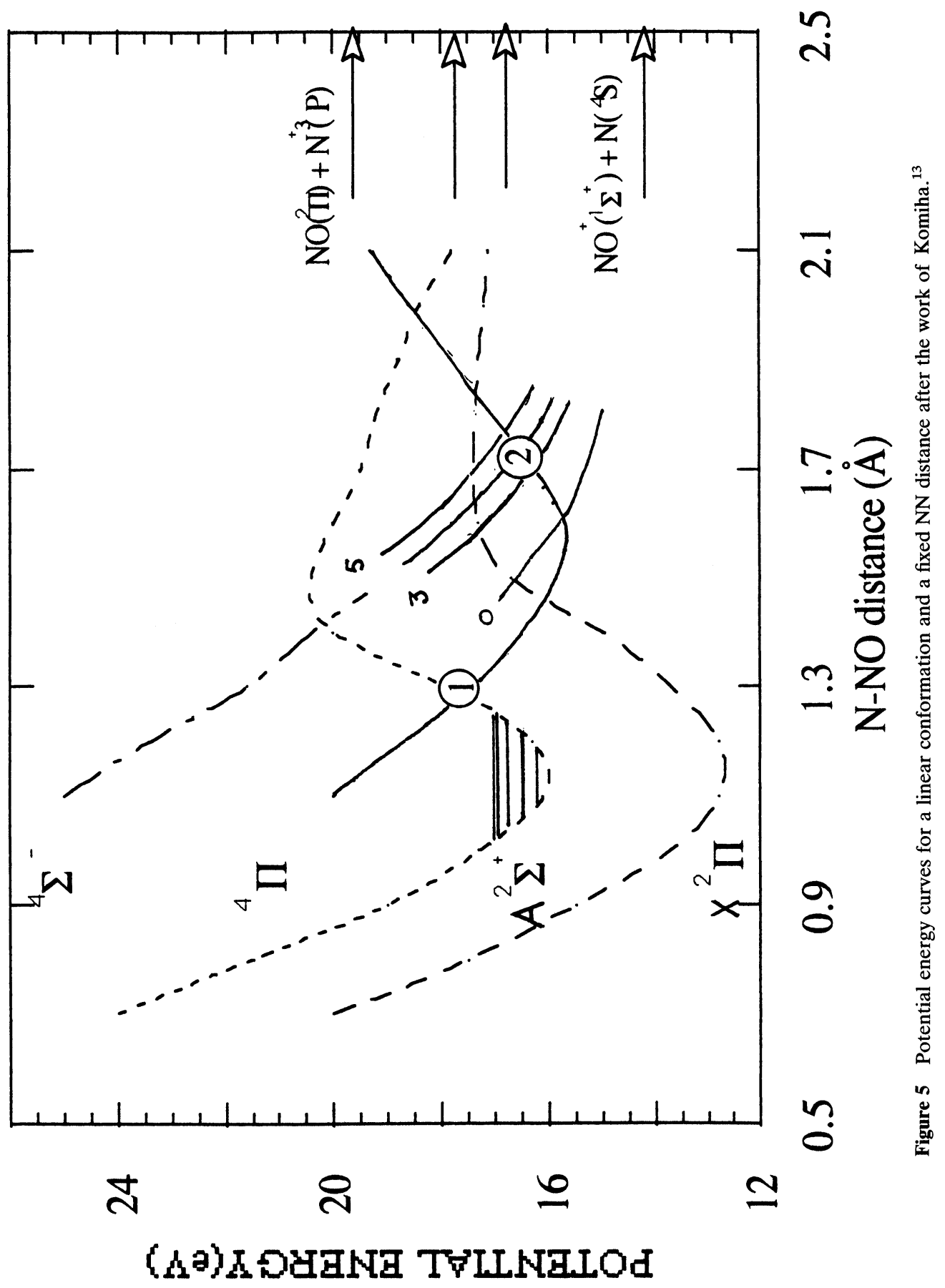


Table $1 \mathrm{O}_{2}^{+}\left(\Omega_{1 / 2}^{+} / \Omega_{3 / 2}^{+}\right)$branching ratio

\begin{tabular}{lll}
\hline$H(0) F 1 \Omega=2$ & $H(0) F 2 \Omega=1$ & $H(0) F 3 \Omega=0$ \\
\hline $0.26 \pm 0.04$ & $1.39 \pm 0.04$ & $4.6 \pm 0.06$
\end{tabular}

$\mathrm{N}-\mathrm{NO}$ reaction coordinate, $\mathrm{NO}$ being fixed at its equilibrium distance. Portions of ${ }^{4} \Sigma$-vibronic potentials correlating to different $\mathrm{NO}^{+}$vibrational states are also shown. The A state does not correlate to either of the two exoergic fragment channels $\mathrm{NO}^{+}\left(\mathrm{X}^{1} \Sigma^{+}\right)+\mathrm{N}\left({ }^{4} \mathrm{~S}\right)$ not to $\mathrm{NO}\left(\mathrm{X}^{2} \Pi\right)+\mathrm{O}^{+}\left({ }^{4} \mathrm{~S}\right)$. Actually the coupling is very weak between the $\mathrm{A}$ state and the repulsive ${ }^{4} \Sigma^{-}$because of negligible Franck-Condon overlap due to the high location of the crossing $n^{\circ} 1$. Because of this, $\mathrm{N}_{2} \mathrm{O}^{+}$A state fluoresces. It is well characterized by fluorescence and photoelectron spectroscopy so that it presents a unique case for the investigation of the role of vibronic state preparation on the dissociation. The first evidence for predissociation was obtained by photoionization mass spectrometry ${ }^{14}$ where $\mathrm{NO}^{+}$was found to be the dominant fragment ion. The observation of radiation from the A state raised the question whether the fragmentation occurred in the ground state, or in the upper A state. The latter was shown to have a stable ground vibrational (000) level and predissociated excited vibrational levels by the pioneering PEPICO experiment of Eland et al. ${ }^{15} \mathrm{In}$ addition it was observed that the $\mathrm{NO}^{+}$fragments were produced in a sharp distribution of internal energy levels. Later Nenner $e t$ al. ${ }^{16}$ in a higher resolution TPEPICO experiment looked at the predissociation of selected vibronic levels and observed a fast rise of the predissociation rate with increasing vibrational energy. This very peculiar dissociation process stimulated new experimental as well as theoretical work. Predissociation rate constants were determined by Maier et.${ }^{17}$ in

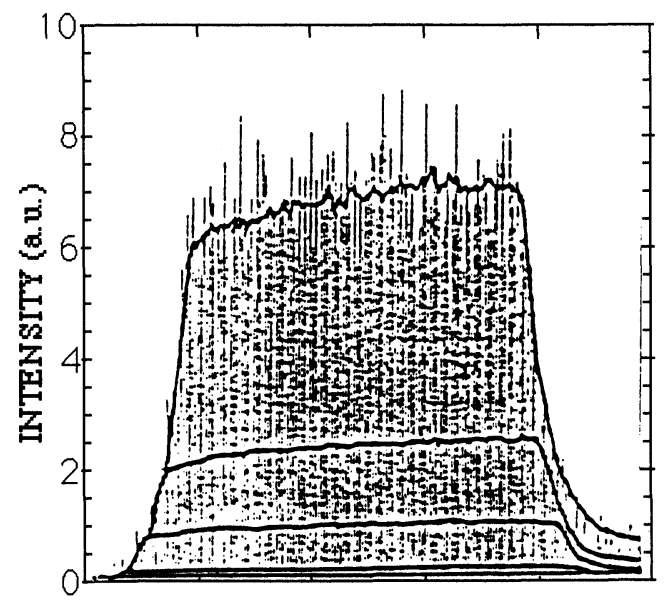

TIME OF FLIGHT

Figure 6 Time of flight distribution of $\mathrm{NO}_{+}$ions formed by predissociation of the $(2,0,0)$ vibronic level of $\mathrm{N}_{2} \mathrm{O}^{+}$A state. 
fluorescence decay measurements using pulsed excitation sources. The results show a strong dependence of the lifetime not only on vibrational energy but also on the type of excited vibrational mode in particular, bending motion is seen to promote dissociation more efficiently than the stretching motion. These lifetime measurements could be rationalized by theory ${ }^{18,19}$ with the assumption that the bound $\mathrm{A}$ ${ }^{2} \Sigma^{+}$was predissociated by a purely repulsive state; bending the molecular ion would lower the crossing $n^{\circ} 1$ and favor predissociation because of a greater nuclear overlap with the A state. This interpretation however left open the origin of the $\mathrm{NO}^{+}$final state distribution which peaked at $v=4$ since a purely repulsive potential for the $\mathrm{N}-\mathrm{NO}^{+}$dissociation channel would produce a distribution of $\mathrm{NO}^{+}$levels peaking at $v=0$. The ab initio calculation of Komiha,${ }^{13}$ the laser induced dissociation of $\mathrm{N}_{2} \mathrm{O}^{+}$ Lermé et al..$^{20}$ and the new TPEPICO experiment of Richard-Viard et al. ${ }^{6}$ in Orsay shown in Figure 6 contributed to a more complete explanation.

The flat top peak for $(2,0,0)$ level shows that the kinetic energy released is distributed among vibrational energy and not rotational energy and that the distribution is isotropic which is likely for a slow predissociation. Then for a fast dissociation i.e. $t<10 \mathrm{~ns}$ the peak shape for each vibrational energy of the fragment
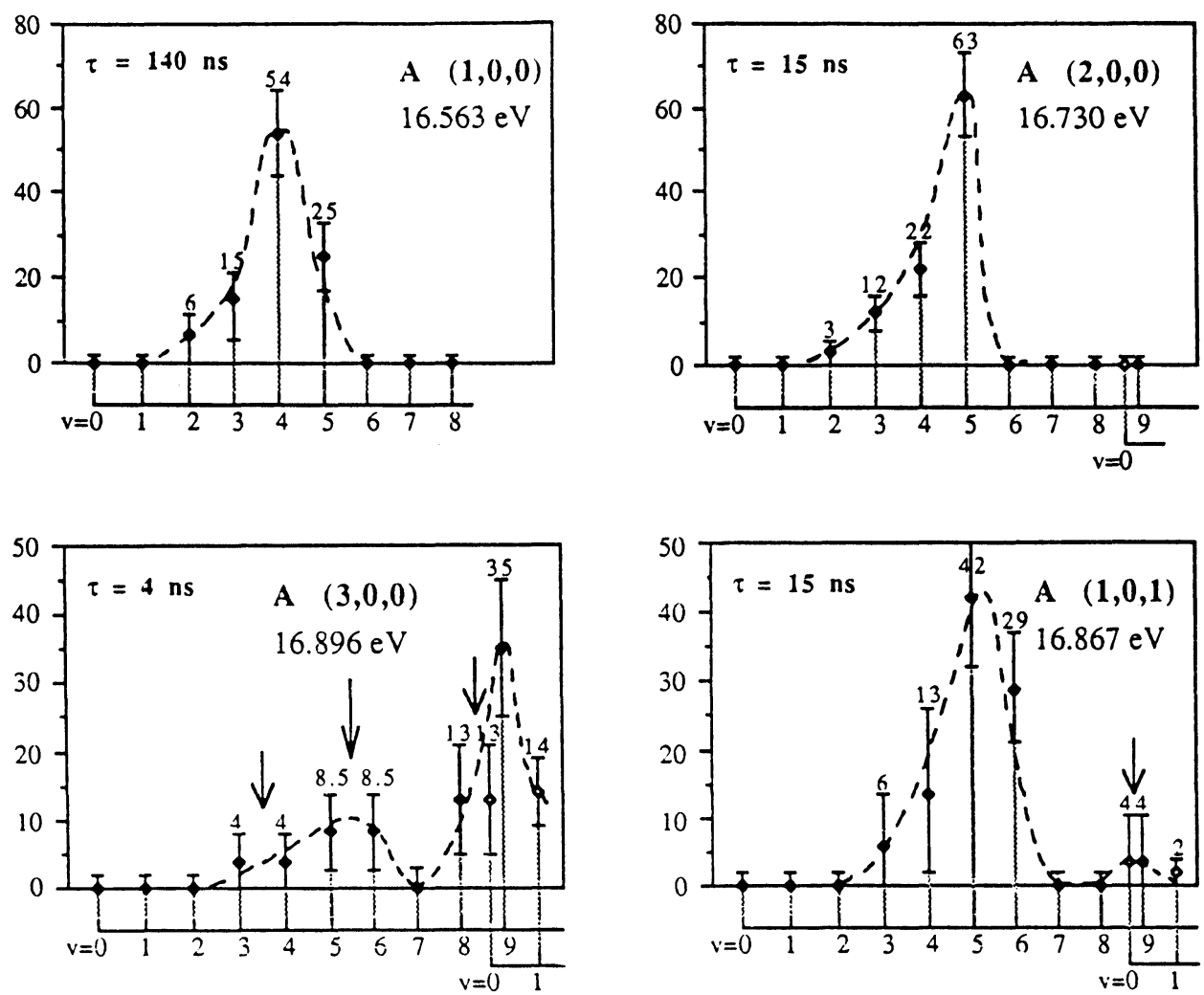

Figure 7 Time of flight distributions of $\mathrm{NO}^{+}$fragments obtained from the predissociation of the $\mathrm{A}(2,0,0)$ level and the results of a Monte-Carlo simulation. 
should be rectangular as observed. The small tailing on both sides of the peak is due to the initial thermal velocity of the molecules in the effusing beam. The asymmetry towards long time of flights is due to the slight metastability $(\tau(2,0,0)=40 \mathrm{~ns})$ of the parent ions. The $\mathrm{NO}^{+}$vibrational energy distributions from various levels are shown in Figure 7. The distributions are very narrow and sharply cut at $v=4(1,0,0)$ and $5(2,0,0)$. Interpretation of the data in the light of the potentials in Figure 5 leads to the following conclusions:

The predissociation of $\mathrm{A}^{2} \Sigma^{+}$is indirect and involves a transition to the intermediate ${ }^{4} \Pi$ state near the crossing $n^{\circ} 1$; this state is in turn predissociated swiftly near the crossing $n^{\circ} 2$ by the ${ }^{4} \Sigma$-repulsive state. The dynamical model proposed by Richard-Viard ${ }^{6}$ gives the basis of a qualitative interpretation of the vibrational population inversion: if we describe the potentials as quasi-diatomic as shown in Figure 5 , a transition at the crossing $n^{\circ} 1$ will populate the ${ }^{4} \Pi$ state with no excitation in the NO coordinate. The transition probability between the ${ }^{4} \Pi$ and ${ }^{4} \Sigma$ - at the crossing $n^{\circ} 2$ is then determined by the Franck-Condon overlap factors between the two states. The sharp cut of the distribution at $v \leqq 6$ is due to the fact that the higher levels crossings lie too high in energy.

This work on the half collision bears interest on the charge transfer reaction of $\mathrm{N}^{+}\left({ }^{3} \mathrm{P}\right)+\mathrm{NO}\left({ }^{2} \Pi v=0\right)$ on the ${ }^{4} \Pi$ surface to produce $\mathrm{N}\left({ }^{4} \mathrm{~S}\right)+\mathrm{NO}^{+}\left({ }^{1} \Sigma^{+}\right)$by a non adiabatic transition to the ${ }^{4} \Sigma$ surface. A very different distribution of final $\mathrm{NO}^{+}$ levels is expected.

\section{References}

1. D. Villarejo, R. Stockbauer and M. G. Inghram, J. Chem. Phys. 48, 3342 (1968) and 50, 4599 (1969).

2. W. B. Peatman, T. B. Borne and E. W. Schlag, Chem. Phys. Lett. 3, 492 (1969) and T. Baer, W. B. Peatman and E. W. Schlag, Chem. Phys. Lett. 4, 243 (1969).

3. R. Spohr, P. M. Guyon, W. A. Chupka and J. Berkowitz, Rev. of Sci. Inst. 42, 1872 (1971).

4. P. M. Guyon, T. Baer, I. Nenner, T. Govers, A. Tabché-Fouhaillé, L. F. A. Ferreira and R. Botter, J. Phys. B. Atom. Molec. Phys. 11, L141 (1978).

5. P. M. Guyon, J. Hepburn, T. Weng, F. Heiser and D. Reynolds, Phys. Rev. Lett. 67, 675 (1991).

6. M. R. Viard, O. Atabek, O. Dutuit and P. M. Guyon, J. Chem. Phys. 93, 8881 (1990).

7. P. M. Guyon, W. A. Chupka, J. Berkowitz, J. Chem. Phys. 65, 1650 (1976).

8. H. Frohlich, P. M. Guyon and M. G. Maujean, J. Chem. Phys. 94, 1102 (1991).

9. P. Natalis, P. Pennetreau, L. Longton and J. E. Collin, J. Electron Spectrosc. Relat. Phenom. 27, 267 (1982).

10. P. M. Dehmer and W. Chupka, J. Chem. Phys. 62, 4525 (1965).

11. P. M. Guyon and L. F. A. Ferreira, Proceedings of the Workshop on some aspects of autoionisation in atoms and small moleucles: Argonne National Laboratory (May 2-3 1985) ANL report ANL-PHY85-3 pp. 129-145.

12. I. Nenner and A. Beswick, Handbook on synchrotron radiation, vol. II, pp. 355, G. V. Marr ed. North Holland.

13. N. Komiha, These 3 eme cycle, Université Pierre et Marie Curie, Paris (1981).

14. V. H. Dibeler, J. A. Walker and S. K. Liston, J.R. Nat. Bur. Stand. 71A, 371 (1967).

15. J. H. D. Eland, Intern. J. Mass. Spectrom. Ion. Phys. 12, 389 (1973).

16. I. Nenner, P. M. Guyon, T. Baer and T. Govers, J. Chem. Phys. 72, 6587 (1980).

17. J. P. Maier and F. Thommen, Chem. Phys. 51, 319 (1980).

18. J. A. Beswick and M. Horani, Chem. Phys. Lett. 78, 4 (1981).

19. S. Miret-Artès, G. Delgado-Bario, O. Atabek and J. A. Beswick, Chem. Phys. 98, 554 (1983).

20. J. Lermé, S. Abed, M. Larzilière, R. A. Holt and M. Carré, J. Chem. Phys. 84, 2167 (1986). 